\title{
Trunk muscle activity during pressure feedback monitoring among individuals with and without chronic low Back pain
}

\author{
Xin Li ${ }^{1 \dagger}$, Wai Leung Ambrose Lo ${ }^{1 \dagger}$, Song-wei Lu', Howe Liu², Ke-yu Lin', Jian-yang Lai', Le Li ${ }^{1 *}$ and \\ Chu-huai Wang ${ }^{1 *}$ (D)
}

\begin{abstract}
Background: Pressure biofeedback unit (PBU) is a widely used non-invasive device to assist core muscle training by providing pressure feedback. The aim this study was to compare the muscle activities of transverse abdominis (TA) and multifidus (MF) at different target pressures $(50,60$ and $70 \mathrm{mmHg})$ of PBU between individuals with and without CLBP.

Methods: Twenty-two patients with chronic LBP (CLBP) and 24 age matched healthy individuals were recruited. Electromyography (EMG) signals were recorded from the TA and MF muscles while the TA and MF were contracted to achieve PBU pressure value of 50,60 and $70 \mathrm{mmHg}$ in random order. The average EMG amplitude (AEMG) of 3 replicate trials was used in the analysis after normalization to \%MVIC. \%MVIC is defined as the mean of the three AEMG divided by the AEMG of MVIC. Two-way ANOVA was performed to assess the effects of groups (healthy and $\mathrm{CLBP}$ ) and the three different target pressures of PBU. Independent sample $t$-test was conducted to compare between the two groups. Spearman's correlation analysis was performed in the CLBP group to determine potential correlations between EMG activity, NPRS and ODI.

Results: The \%MVIC of the TA and MF in the CLBP group were higher than the control group at each pressure value $(P<0.05)$. During maximal voluntary isometric contraction $(\mathrm{MVIC})$ of TA and $\mathrm{MF}$, compared with healthy groups, CLBP subjects showed a decrease (TA mean $=47.61 \mu \mathrm{V}$; MF mean $=42.40 \mu \mathrm{V}$ ) in EMG amplitudes $(P \leq 0.001)$. The MVIC of MF was negatively correlated with Numerical Pain Rating Scale $(r=-0.48, P=0.024)$ and Oswestry Disability Index $(r=-0.59, P=0.004)$.

Conclusions: We measured the trunk muscles activities at different PBU pressure values, which allows the individual to estimate trunk muscle contraction via PBU. Clinicians may be able to confer the data obtained through EMG recordings to adjust the exercise intensity of PBU training accordingly.
\end{abstract}

Keywords: Low back pain, Multifidus, Pressure biofeedback unit, Transverse abdominals

\footnotetext{
* Correspondence: lile5@mail.sysu.edu.cn; wangchuh@mail.sysu.edu.cn

${ }^{+}$Xin Li and Wai Leung Ambrose Lo contributed equally to this work.

'Department of Rehabilitation Medicine, The First Affiliated Hospital, Sun Yat-sen University, Guangzhou 510080, China

Full list of author information is available at the end of the article
}

(c) The Author(s). 2020 Open Access This article is licensed under a Creative Commons Attribution 4.0 International License, which permits use, sharing, adaptation, distribution and reproduction in any medium or format, as long as you give appropriate credit to the original author(s) and the source, provide a link to the Creative Commons licence, and indicate if changes were made. The images or other third party material in this article are included in the article's Creative Commons licence, unless indicated otherwise in a credit line to the material. If material is not included in the article's Creative Commons licence and your intended use is not permitted by statutory regulation or exceeds the permitted use, you will need to obtain permission directly from the copyright holder. To view a copy of this licence, visit http://creativecommons.org/licenses/by/4.0/. The Creative Commons Public Domain Dedication waiver (http://creativecommons.org/publicdomain/zero/1.0/) applies to the data made available in this article, unless otherwise stated in a credit line to the data. 


\section{Background}

Low back pain (LBP) is a major contributor to global burden of disability [1]. Animal experiment and mathematical model of the spine indicated that sequential injuries and deep muscles weakness resulted in spinal instability [2], which is defined as a significant decrease in the capacity of the spinal stabilizing system to maintain the intervertebral neutral zone within physiological limits [3]. Clinical guidelines suggested that spinal stabilization exercises (SSEs) may be as effective as other physiotherapy treatments in reducing disability and pain $[4,5]$. The principle of SSEs is to train the co-contraction pattern of the deep local trunk muscles of the transversus abdominis (TA) and the multifidus muscles (MF). Despite the popularity to train deep muscles co-contraction to improve spinal stability and pain reduction, this theory remains controversial as fundamental evidence is lacking [6]. Early literature reported that the intrinsic muscle stiffness was not sufficient to stabilize the spine without neuromuscular reflex response (such as under a complex muscle synergy scenario) [6], which is accounted for $42 \%$ of the total stabilizing trunk stiffness [7]. In addition, some scholars suggested that it was the intra-abdominal pressurization, rather than the force activation of the selected abdominal muscles, that contribute to additional lumbar stability [8]. Ultrasonography study also indicated that delay onset of the abdominal muscles was not always present in people with nonspecific LBP [9]. Thus, there also is a lack of uniformity regarding the meaning of "spinal stabilization" and what therapeutic exercises may be most effective [6-8].

The quantitative measurement tool of MF and TA plays a crucial role in assessing muscle activation pattern and clinical effectiveness of SSEs. The gold-standard to measure the activity of deep local trunk muscles is by fine-wire electromyography $[7,8]$. However, factors such as pain, discomfort and risk of infection limit its clinical application as routine outcome measure. Indirect measurements of MF and TA muscles functions rely on electromyography (EMG) and ultrasound imaging. Surface EMG also has the limitation of cross-talk with other muscles that are in close proximity [8]. High cost and inconvenient hinders the common use of ultrasound imaging in clinical practice and the assessment of ultrasound is often limited by the position of the subject [10]. In clinical and research setting, $\mathrm{PBU}$ is a non-invasive, low-cost and convenient to use device that has been used to monitor the change of pressure as a mean to estimate the muscle activation of the MF and TA muscles during specific maneuverer [10, 11]. An inability to maintain the required pressure while performing the posture is reflective of an inability to maintain abdominal muscle contraction, resulting in uncontrolled movement and instability of the lumbar spine [12]. The validity of such approach was to investigate in early literature which reported moderate correlation between changes and PBU pressure and EMG activities [13]. The inter- and intra-examiner reproducibility of PBU in measuring TA muscle activity in people with cLBP [14] and healthy [11] individuals was reported to be excellent.

Previous studies suggested that the pretrial pressure of $70 \mathrm{mmHg}$ was suitable for prone position [10, 14] and $40 \mathrm{mmHg}$ was suitable for hook-lying supine [11], sidelying [15] and upright positions [11]. However, few studies were conducted in seated position. In Australia, 70\% of adults sit for more than $8 \mathrm{~h}$ per day [16], and the time is likely to extend due to the increasing use of social media [17]. Prolonged sitting seems to be unavoidable in our modern daily life, work and study. Training the TA and MF muscles with PBU in the correct seated position could increase lumbar stability, which might be one of the measures to reduce the occurrence of LBP $[11,18]$. To improve the clinical application of PBU and facilitate interpretation of activation of deep local trunk muscles, further investigations are needed to establish the response between activity of trunk muscles and different pressures of PBU in seated position.

This study aimed to answer the research question of whether changes in PBU pressure measured during MF and TA contraction in a seated position may induce corresponding changes in EMG muscle activities. The study also investigated if people with cLBP have different muscle activation pattern of TA and MF in a seated position, and whether changes in muscle activation pattern is associated with pain. We hypothesized that muscle activation patterns were significantly different between people with cLBP and heathy individuals, and the differences in activation pattern were associated with pain and disability.

\section{Method}

\section{Subjects}

Subjects were recruited from the local rehabilitation ward and outpatient department of the hospital. The inclusion criteria of cLBP subjects were as follows: 1) experienced pain in the low back region with or without accompanying buttock pain over the past 3 months, and of sufficient intensity to have limited activities of daily living [14]; 2) pain score range between 3 and 7 on the Numerical Pain Rating Scale (NPRS) [18]; and 3) able to perform the experiment procedure without symptom aggravation. This was to minimize the variability in the level of pain during testing which may increase the variability of the data. Exclusion criteria were: 1) existence of respiratory, orthopaedic, circulatory or neurological conditions; 2) previous surgery to the abdomen or lower back; 3) female subjects who were pregnant or suffered from dysmenorrhea; 4) epilepsy or had family history of epilepsy. The exclusion criteria of NPRS higher than 7 was due to published literature indicated patients who have NPRS 7 or above were not able to perform 
maximum contraction [14, 18]. As maximum contraction was necessary in this study to obtain the MVIC EMG data, this exclusion criteria was adopted. Age matched healthy individuals with no existing LBP and no LBP in the past year were recruited as control.

\section{Ethics}

The study protocol was reviewed and approved by the Human Subjects Ethics Sub-committee of the First Affiliated Hospital, Sun Yat-sen University (Approval number: [2017] C-034). All subjects gave written informed consent. The study was conducted in accordance to the Declaration of Helsinki.

\section{Instruments}

The Pressure Biofeedback Unit (PBU) (Chattanooga Group Inc., LLC Vista, California, USA) employed in this study is made up of a three-chamber air-filled pressure cell, a catheter and a sphygmomanometer gauge. The pressure cell of the PBU was made from latex-free rubber material, and the unfolded dimension of the cell was $16.7 \times 24.0 \mathrm{~cm}$. The sphygmomanometer was calibrated to $2 \mathrm{mmHg}$ interval and has a range between 0 to $200 \mathrm{mmHg}$. Movement or change in position causes volume change in the pressure cells that is displayed on the gauge. Prior to data recording, the pressure cell was first inflated to a pressure of $40 \mathrm{mmHg}$ (orange band). The valve was then closed to stop air leakage [11]. To ensure accuracy of the PBU measurements, the device was pretested by loading a static weight of $4 \mathrm{~kg}$ for $24 \mathrm{~h}$. The PBU was only considered adequate if the device lost no more than $0.5 \mathrm{mmHg}$ during the 24-h period [19].

Muscle activity was assessed by the gold standard surface EMG (UMI-SE-I sEMG system, Shaoxing United Medical Instruments Co., LTD, China). EMG was adopted to study the activity of $\operatorname{TrA}$ due to it being regarded as the gold standard to measure muscle activities, despite its limitation of cross-talk with muscle fibers that are in close proximity. Ultrasonography was not suitable for the present study as the ultrasound probe could not be placed on the location that was required to scan the TrA in a seated position. EMG signals with common mode rejection ratio of $110 \mathrm{db}$, bandwidth of $15-1000 \mathrm{~Hz}$ and resolution of $0.1 \mu \mathrm{V}$. Sampling frequency was set at $3000 \mathrm{~Hz}$ and stored in a computer for offline analysis [18]. To reduce skin impedance, hair was removed from the measurement sites and the skin was deterged with alcohol before electrode placement. Disposable $\mathrm{Ag} / \mathrm{AgCl}$ surface electrodes were attached to the concerned muscle. The maximum space between the recording electrodes was $2 \mathrm{~cm}$. The locations of the EMG electrodes were determined in accordance to EMG placement guidelines [20] and published studies [6, 7, 14]. Electrodes to measure TA activity were placed at the center position that was $2 \mathrm{~cm}$ cephalic to the pubic bone, just lateral to the midline, and parallel to the superior pubic ramus along either side of the course of the underlying muscle fibers [14, 20]. For MF, electrodes were placed at the level of the L5 spinous process along the line joining the posterosuperior iliac spine (PSIS) and L1-L2 vertebral interspace $[18,20]$. To obtain the MVIC data of the TA, subjects were in sit-up styled movements with both legs fixated on the floor, using a belt or manually fixed. The spine then flexed to $30^{\circ}$ and maintained the position for $5 \mathrm{~s}$ to record MVIC [20]. To obtain the MVIC data of MF, the subjects laid in prone position on a standard plinth with the upper limbs positioned overhead. Subjects then lifted their head and upper extremities with maximum effort and maintained the position for $5 \mathrm{~s}$ to record MVIC $[18,20]$. The maximal voluntary isometric contraction (MVIC) was measured to determine the level of voluntary contractions during testing. MVIC was repeated 3 times and the highest value was selected for analysis $[11,20]$. The average EMG amplitude (AEMG) at each pressure level was obtained from 3 repeated trials. AEMG was used in the data analysis after normalization to \%MVIC [11, 20], which was calculated for the selected muscles using the formula: \% MVIC $=\frac{A E M G}{M V I C} \times 100 \%$. The slope of the \%MVIC-pressure relation was calculated at $70 \mathrm{mmHg}$ and $50 \mathrm{mmHg}$ pressure and compared between the two groups. The rationale to choose AEMG and \%MVIC as variables was that raw EMG readings were not comparable between individuals. By normalizing the MVC, the raw EMG reading was rescaled to a percentage of a reference value that was standardized across all individuals within the study. The standardized MVC values enabled quantitative comparisons of the EMG readings between individuals.

\section{Experimental procedures}

Demographics and background clinical information were first collected. Participants were asked to score the pain intensity over the last $24 \mathrm{~h}$ on the NPRS. The scale range between 0 and 10, with 0 being no pain and 10 being severe pain [21]. Pain related disability was assessed by the Chinese version of the Oswestry Disability Index (ODI) [22]. The total score of ODI ranges from 0 to 100, with 0 being no disability and 100 being maximum disability [22].

All subjects then received information about the anatomy, biomechanics and functions of the TA and MF muscles. All subjects were asked to fast for at least $2 \mathrm{~h}$, avoid performing any type of abdominal exercises, and empty their bladders prior to data collection [10]. The procedures of PBU tests were described in Table 1. They were performed in random order by selecting a single card with three cards marked with 1 (50 
Table 1 Methods for positions of interest muscles and EMG signals

\begin{tabular}{ll}
\hline & Description of the methods \\
\hline Transversus & Subjects were seated upright against \\
Abdominis & the wall with one hand holding the \\
(TA) & bothymomanometer gauge and \\
pressure cell was placed behind the \\
lumbar spine. Subjects slowly pulled \\
their lower abdomen and navel \\
towards the back to contract TA \\
without changing spinal position \\
change [11, 14]. The PBU pressure \\
of 50 mmHg, 60mmHg and 70mmHg \\
were selected in random \\
order. \\
Subjects were seated uprightly \\
against the wall with one hand \\
holding the sphygmomanometer \\
gauge and both feet rested on the \\
floor. The pressure cell was placed \\
behind the medial edges of the \\
shoulder blades. Subjects slowly \\
extended the lumbar spine to \\
contract MF [18, 20], until the PBU \\
pressure reached 50 mmHg, \\
60mmHg or 70 mmHg. \\
(MF)
\end{tabular}

$\mathrm{mmHg}), 2(60 \mathrm{mmHg})$, or $3(70 \mathrm{mmHg})$. The examiner provided verbal instructions throughout the test. Subjects were asked to maintain within $\pm 2 \mathrm{mmHg}$ from the target pressure [11]. The accuracy of maintaining the required PBU pressure was confirmed by visual inspection of the gauge.

All subjects in the pain-free group selected the muscles on the right side. Subjects in the cLBP group chose the more painful side as target muscles. Three trials were conducted for each target pressure and the averaged values were used for analysis. A resting interval of $30 \mathrm{~s}$ was provided between each trial to minimize fatigue. Participants were allowed to stand up and move during the interval.

\section{Statistical analysis}

Statistical analysis was conducted in the statistical Package for the Social Sciences version 25.0 software (SPSS Inc., Chicago, IL, USA). The EMG data at different PBU pressures were expressed as mean and standard deviation. The Shapiro-Wilk test was used to test the normality distribution of the data. Wilcoxon's signed-rank test was used for non-normal distribution parameters. Independent sample t-test was conducted to compare subject characteristics. Two-way ANOVA was performed to assess the effects of groups (Healthy and cLBP) and the three different target pressures (50, 60 and $70 \mathrm{mmHg}$ ) of PBU. If the main effect of the pressure was significant, a post hoc test was performed using the Bonferroni correction. If the main effect of the groups was significant, independent sample $t$-test was conducted to compare between two groups. Spearman's correlation analysis was performed in the cLBP group to determine the correlations between EMG activity, NPRS and ODI. A value of $P<0.05$ was considered to be statistically significant.

\section{Results}

Twenty-two right-handed individuals were recruited in cLBP group and 24 age matched healthy individuals were recruited in the control group. The sample characteristics of both cohorts are presented in Table 2. There was no group difference for gender, age, height, weight, BMI and educational level. Two cLBP individuals were unable to maintain $70 \mathrm{mmHg}$ of TA and were excluded from the analysis. Twenty cLBP participants were included in the analyses of TA at the pressure value of 70 mmHg (Table 3).

Table 2 Characteristics of the sample cohorts (mean (SD))

\begin{tabular}{llll}
\hline Demographics & CLBP $(n=22)$ & Pain-free controls $(n=24)$ & $P$-value \\
\hline Gender (M: F) & $6: 16$ & $5: 19$ & 0.613 \\
Age (years) & $28.27(8.15)$ & $25.17(4.00)$ & 0.103 \\
Height (cm) & $163.55(9.22)$ & $163.88(7.24)$ & 0.893 \\
Weight (kg) & $57.86(7.47)$ & $54.92(6.56)$ & 0.277 \\
BMI (kg/m $\left.{ }^{2}\right)$ & $21.59(1.78)$ & $20.25(1.79)$ & $15.58(0.78)$ \\
Education level (years) & $15.36(2.52)$ & - & 0.059 \\
Side of pain (L: R) & $9: 13$ & - & - \\
Pain intensity (NPRS) & $4.73(1.45)$ & - & - \\
Pain duration (years) & $3.65(5.33)$ & - & - \\
ODI (\%) & $25.64(11.85)$ & -
\end{tabular}

Key: CLBP chronic Low Back Pain, BMI body mass index, ODI Oswestry disability index, $L$ left, $R$ right, NPRS numerical pain rating scale, SD standard deviation 
Table 3 At each target pressure value, mean and standard deviation (SD) of AEMG $(\mu V)$ and MVIC for TA and MF muscles

\begin{tabular}{|c|c|c|c|}
\hline Interest muscles at target pressure value & $\operatorname{cLBP}(n=22)$ & Pain-free controls $(n=24)$ & $P$ \\
\hline \multicolumn{4}{|l|}{ Transversus Abdominis (TA) } \\
\hline MVIC & $49.44(22.98)$ & $97.05(55.09)$ & $<0.001$ \\
\hline $50 \mathrm{mmHg}$ & $13.75(12.41)$ & $11.60(8.88)$ & 0.792 \\
\hline $60 \mathrm{mmHg}$ & $18.85(13.93)$ & $16.16(13.66)$ & 0.468 \\
\hline $70 \mathrm{mmHg}(n=20 / 22)$ & $23.38(16.01)$ & $19.58(14.84)$ & 0.437 \\
\hline \multicolumn{4}{|l|}{ Multifidus (MF) } \\
\hline MVIC & $61.38(37.21)$ & $103.78(42.29)$ & 0.001 \\
\hline $50 \mathrm{mmHg}$ & $11.55(7.91)$ & $11.19(7.59)$ & 0.886 \\
\hline $60 \mathrm{mmHg}$ & $17.94(9.63)$ & $18.16(12.3)$ & 0.843 \\
\hline $70 \mathrm{mmHg}$ & $26.60(13.36)$ & $27.86(18.23)$ & 0.912 \\
\hline
\end{tabular}

Key: CLBP chronic Low Back Pain, MF multifidus, MVIC maximal voluntary isometric contraction, $T A$ transversus abdominis

\%MVIC at different pressure values of PBU training

Figure 1 shows the response between $\mathrm{TA}$ and $\mathrm{MF}$ muscle activities at different target pressures of PBU, and the differences in TA and MF between individuals with and without cLBP. For TA, the results of two-way ANOVA tests indicated no statistically significant difference among the two groups and different pressures ( $F$ interaction-effect $=0.825, P=0.440)$, the main effect of the pressure was not significant either $(F$ pressure-main $=$ $0.198, P=0.821)$, and the groups effect was significant $(F$ group-main $=4.500, P=0.036)$. Similar results were found in MF $(F$ interaction-effect $=0.713, P=0.492 ; F$ pressure-main $=0.071, \quad P=0.932 ; \quad F$ group-main $=7.569, \quad P=$
0.007). The \%MVIC of the TA and MF in the cLBP group were statistically higher than the control group at every pressure value $(P<0.05)$. In both groups, the $\%$ MVIC of TA were more active than the MF at 50 $\mathrm{mmHg}$ and $60 \mathrm{mmHg}$. At $70 \mathrm{mmHg}$, the cLBP group had almost equal activity in both muscles, while the MF $\%$ MVIC of the healthy group had higher activity than TA \%MVIC.

Both the TA $(F=3.721, P=0.03)$ and the $\mathrm{MF}(F=5.86$, $P=0.005)$ \%MVIC of cLBP group showed significant differences between $50 \mathrm{mmHg}$ and $70 \mathrm{mmHg}$. In the painfree group, there were statistically significant differences in MF \%MVIC under each of the three pressure $(F=$

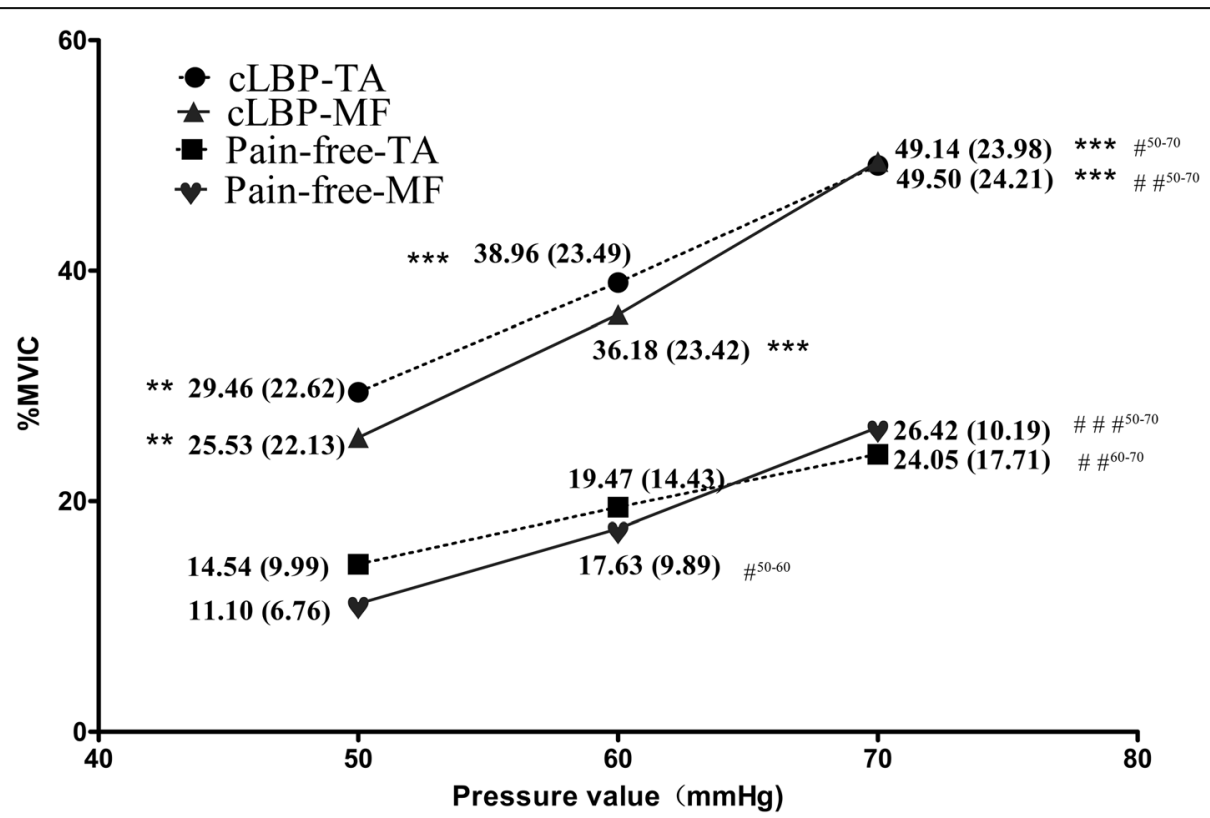

Fig. 1 The association between deep local trunk muscles (TA or MF) and different target pressures of PBU at seating position, and the different for the local trunk muscles (TA or MF) between individuals with and without CLBP. CLBP, chronic Low Back Pain; EMG, electromyography; MF, Multifidus; MVIC, maximal voluntary isometric contraction; TA, Transversus Abdominis; * indicates the difference between CLBP group and the

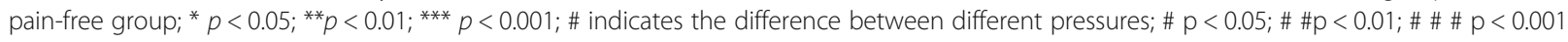


17.202, $P<0.001)$, while there was no difference in TA \%MVIC under each pressure $(F=1.131, P=0.329)$.

\section{Average EMG amplitudes (AEMG)}

During MVIC of TA and MF, cLBP group were significantly less than the healthy group $(P \leq 0.001)$. No difference was observed in the AEMG at any target pressure value $(P>0.05)$. Table 3 illustrates the mean and standard deviation of AEMG and MVIC for the TA and the MF at each target pressure.

\section{Correlation between EMG activity, NPRS and ODI}

MF MVIC was negatively correlated with NPRS $(r=-$ $0.48, P=0.024)$ (Fig. 2a) and ODI $(r=-0.59, P=0.004)$ (Fig. 2b). No significant correlation between TA MVIC and NPRS $(r=-0.12, P=0.591)$, or between TA MVIC and ODI $(r=-0.26, P=0.250)$ were observed. Both of the MF MVIC and TA MVIC were not significantly correlated with pain duration. Other EMG activities (\%MVIC and AEMG of TA, MF at any target pressure) were not significantly correlated with NPRS, ODI and pain duration. ODI was positively correlated with NPRS $(r=0.56, P=0.007)$ (Fig. 2c) and pain duration $(r=0.52$, $P=0.014$ ) (Fig. 2d). Figure 2 illustrates the correlation between EMG activity, NPRS and ODI.

\section{Discussion}

The present study observed that PBU measurement during MF and TA contraction in a seated position induced corresponding changes in EMG muscle activities. Muscle activities of the TA and MF during MVIC were lower in the cLBP group than the control group. This indicated that people with cLBP were unable to generate as much force as healthy individuals. However, muscle activities were significantly higher at each pressure value in the cLBP group than the control group. This indicated a stronger contraction was required to maintain spinal stability in people with cLBP. The MVIC of MF was negatively correlated with NPRS and ODI. TA MVIC was not correlated with NPRS or ODI.
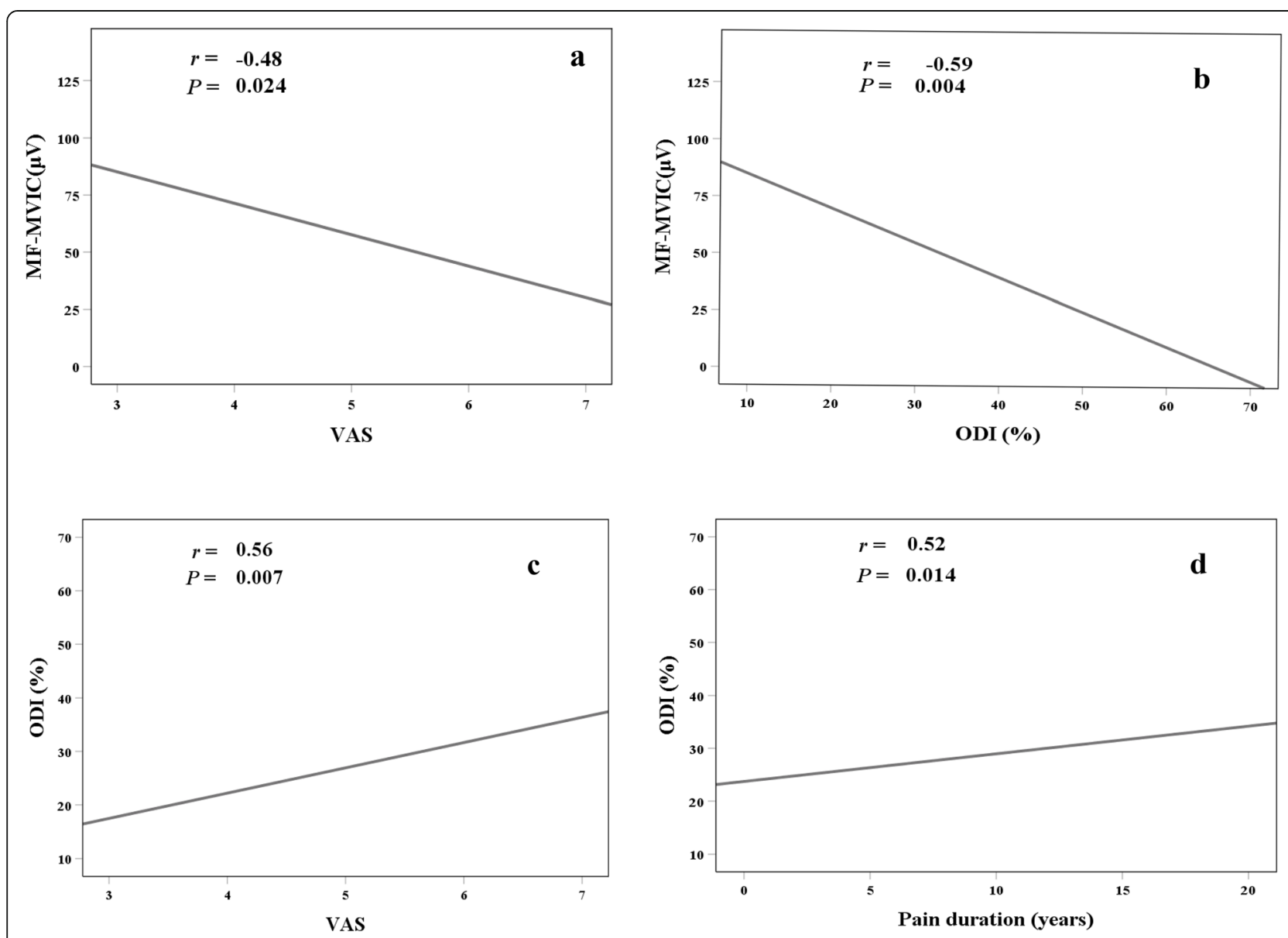

Fig. 2 Correlation between EMG activity and NPRS, ODI. CLBP, chronic Low Back Pain; MF, Multifidus; MVIC, maximal voluntary isometric contraction; ODI, Oswestry Disability Index; TA, Transversus Abdominis; NPRS, Numerical Pain Rating Scale 


\section{MF and TA muscle activities in healthy controls vs CLBP subjects}

Our results indicated that the \%MVIC of the TA and MF in the CLBP group were statistically higher than the control group at every pressure value. For MF, finding of our present study was consistent with previous research published by Ansari et al. [23] which explained the high $\%$ MVIC of MF. They suggested that muscle pain could be accompanied by hyperactivity in the back muscles during dynamic conditions, which was known as the pain adaptation model [23]. The significantly reduced MVIC of the MF muscle in cLBP group might also support this phenomenon. However, the mechanism of MVIC reduction is not totally understood, but may be related to pain inhibition which limits the ability to perform maximum muscle contraction. People with cLBP have a greater sensitivity to pain [24]. Thus, we speculate that cLBP alters spontaneous neuronal activity resulting in changes in EMG activity [18]. Additionally, atrophic changes of MF had been confirmed in around 77-80\% of LBP cases, especially at the L5-S1 level [25] (the EMG site of MF in our study), which might contribute to lower MVIC in the cLBP group.

TA is an important deep muscle that plays a key role in the dynamic control of the lumbar spine $[6,18]$. The present study observed lower TA EMG amplitudes during MVIC in the LBP group than the healthy group. Hodge et al. reported a delay in TA muscle contraction relative to the agonist muscle that moved the limbs in people with LBP [26]. Gildea et al. [27] reported that under contraction status, the thickness of TA was higher in female dancers with cLBP than those without pain. These data support our finding of low TA muscle activity during MVIC.

\section{Differences in muscle activation pattern}

Our results showed that the TA \%MVIC was more active than MF \%MVIC at $50 \mathrm{mmHg}$ and $60 \mathrm{mmHg}$ in both groups. These findings are consistent with published studies that investigated the relationship between TA and MF in patients with cLBP [28]. The study reported that patients who had adequate contraction of multifidus were of 4.5 times likely to be able to contract TA. At the PBU pressure of $70 \mathrm{mmHg}$, the cLBP group demonstrated almost equal muscle activity in both TA and MF muscles, whereas in the healthy group the \%MVIC of MF had more activity than TA. The potential reason may be related to the fatigue of the multifidus muscle. According to published literature [25, 29], sensorial factors influence the recruitment of TA and contributed to MF fatigue. The study by Ramos et al. [30] utilized surface EMG to assess fatigue of MF and PBU to detect activity of TA in patients with LBP. They reported that patients with LBP had difficulties to depress the abdominal wall at the PBU pressure $70 \mathrm{mmHg}$ and higher. MF fatigue was also observed. Another possible reason was that cLBP patients had reduced flexibility and mobility in the frontal, transverse, and sagittal planes of motion [31]. When TA was contracted at 70 $\mathrm{mmHg}$, there was limited space in the anatomical position [31] that the low back required to complete the motion.

\section{Correlation between muscle activities, pain and disability} The present study observed a negative correlation between MVIC of MF and NPRS and ODI which was consistent with previous studies [18]. There was no correlation between the MVIC of TA and NPRS or ODI. The potential reason was that TA and MF have different roles in maintaining lumbar stability due to their different anatomical structures, different muscle fiber size, different motor unit control properties [24, 25]. Previous studies suggested that the TA was mainly involved in lumbar stability by contractile increase of abdominal pressure $[10,14]$, and MF directly maintain lumbar stability through the thoracolumbar fascia [5, 24, 32]. Therefore, compared with the TA, MF might be more correlated with NPRS and ODI. Moreover, TA and MF had different neuromuscular and proprioceptive systems, along with varied changes in biomechanical alignment of the spine and developed different models of pain adaptation [33]. However, speculation on the connection between neuromuscular control mechanisms and pain was difficult as little is known about the underlying relationship between brain network and the TA and MF muscle activity [28].

\section{Limitations}

First, the application of PBU to indirectly estimate muscle activity is a limitation. The measurement of pressure simply indicates whether an individual is able to maintain a constant pressure while performing a particular activity. It is inconclusive if the measurement properties of PBU for the assessment of TA activity is adequate. Therefore, PBU measurement should not be used solely as a mean to estimate muscle activity. Surface EMG measured muscle activities has the limitation of cross-talk from muscles that are in close proximity. Although ultrasonography does not have such limitation and may be more appropriate to study TA activity, however, it was not technical feasible to perform ultrasound scan of the TA in a seated position. The reliability and validity of the PBU and EMG in measuring MF muscle activity in patients with cLBP were not established. Further studies are needed to assess the reliability and validity of this method for evaluating MF. Second, cLBP may interfere with the person's ability to perform maximum muscle contraction. Individuals may therefore not able 
to perform their "maximum" ability during MVIC due to pain aggravation. The assessor provided detail instructions and verbal encouragements in the present study as an attempt to minimize the impact. The uneven distribution of gender in the sample population might be also a limitation.

\section{Conclusions}

We measured the trunk muscles activities at different PBU pressure values, which allows the individual to estimate trunk muscle contraction via PBU. Clinicians may be able to confer the data obtained through EMG recordings to adjust the exercise intensity of PBU training accordingly.

\section{Abbreviations}

BMI: Body mass index; CLBP: chronic low back pain; EMG: Electromyography; ICC: Intraclass correlation coefficient; MF: Multifidus; MVIC: Maximal voluntary isometric contraction; ODI: Oswestry disability index; PBU: Pressure biofeedback unit; PSIS: Posterosuperior iliac spine; SD: Standard deviation; SSES: Segmental spinal stabilization exercises; TA: Transversus abdominis; NPRS: Numerical pain rating scale

\section{Acknowledgements}

The authors thank the participants and Yan Tang for their contribution to the study. We also thank our friend Meng Zhao for her help with the two pictures in the Table 1.

\section{Authors' contributions}

All authors have read and approved the final manuscript. All authors meet the four primary ICMJE criteria for authorship. In addition, all authors have been actively involved in the study in different capacities: XL designed the study and conducted all stages of the study including data collection, analysis, interpretation, and drafting of the manuscript. SL, KLand JL participated in the recruitment and data analysis. HL revised the manuscript. $\mathrm{LL}$ and WLAL revised the manuscript, interpreted the data and managed the trial. CW contributed to study conception and design, data interpretation, and revising the manuscript.

\section{Funding}

This work was supported by the National Natural Science Foundation of China under Grant [number 81772434] and the Special Fund for Science and Technology of the Industry-University-Research Cooperative Innovation of Guangzhou, China, under Grant [number 201704020122]. The funding bodies provided support for the trial and publication of the present manuscript. The funding bodies did not influence the design of the study and collection, analysis, and interpretation of data and in the writing of the manuscript.

\section{Availability of data and materials}

The dataset supporting the conclusions of this article is available from the authors upon request.

\section{Ethics approval and consent to participate}

The study protocol was reviewed and approved by the Human Subjects Ethics Sub-committee of the First Affiliated Hospital, Sun Yat-sen University (Approval number: [2017] C-034). All subjects gave written informed consent. The study was conducted in accordance to the Declaration of Helsinki.

\section{Consent for publication}

Written informed consent was obtained from all participants for the publication of the data present in the manuscript.

\section{Competing interests}

The authors declare that they have no competing interests. The authors have no financial relationship with the manufacturer of the used in the present study.

\section{Author details}

'Department of Rehabilitation Medicine, The First Affiliated Hospital, Sun Yat-sen University, Guangzhou 510080, China. 'Department of Physical Therapy, University of North Texas Health Science Center, Fort Worth, TX 76101, USA

Received: 10 December 2019 Accepted: 3 August 2020

Published online: 22 August 2020

\section{References}

1. Clark S, Horton R. Low Back pain: a major global challenge. Lancet. 2018; 391(10137):2302

2. Shi C, Qiu S, Riester SM, Das V, Zhu B, Wallace AA, et al. Animal models for studying the etiology and treatment of low back pain. J Orthop Res. 2018; 36(5):1305-12

3. Panjabi MM. The stabilizing system of the spine. Part II. Neutral zone and instability hypothesis. J Spinal Disord. 1992;5(4):390-7.

4. Malfliet A, Ickmans K, Huysmans E, Coppieters I, Willaert W, Bogaert W, et al. Best Evidence Rehabilitation for Chronic Pain Part 3: Low BackPain. J Clin Med. 2019;8:1-24.

5. Rackwitz B, de Bie R, Limm H, von Garnier K, Ewert T, Stucki G. Segmental stabilizing exercises and low back pain. What is the evidence? A systematic review of randomized controlled trials. Clin Rehabil. 2006;20(7):553-67.

6. Allison G, Morris S. Transversus abdominis and core stability: has the pendulum swung? Br J Sports Med. 2008;42(11):930-1.

7. Moorhouse KM, Granata KP. Role of reflex dynamics in spinal stability: intrinsic muscle stiffness alone is insufficient for stability. J Biomech. 2007; 40(5):1058-65.

8. Stokes I, Gardner-Morse M, Henry S. Abdominal muscle activation increases lumbar spinal stability: analysis of contributions of different muscle groups. Clin Biomech. 2011;26(8):797-803.

9. Gubler D, Mannion AF, Schenk P, Gorelick M, Helbling D, Gerber H, et al. Ultrasound tissue Doppler imaging reveals no delay in abdominal muscle feed-forward activity during rapid arm movements in patients with chronic low back pain. Spine (Phila Pa 1976). 2010;35(16):1506-13.

10. da Silva AP, Dos Santos RPM, Coertjens PC, Coertjens M. Clinimetric properties of the pressure biofeedback unit method for estimating respiratory pressures. Physiother Theory Pract. 2017;33(4):345-51.

11. Jung DE, Kim K, Lee SK. Comparison of muscle activities using a pressure biofeedback unit during abdominal muscle training performed by Normal adults in the standing and supine positions. J Phys Ther Sci. 2014;26(2):191-3.

12. Cynn HS, Oh JS, Kwon OY, Yi CH. Effects of lumbar stabilization using a pressure biofeedback unit on muscle activity and lateral pelvic tilt during hip abduction in Sidelying. Arch Phys Med Rehabil. 2006;87(11):1454-8.

13. Hodges P, Richardson C, Jull G. Evaluation of the relationship between laboratory and clinical tests of transversus abdominis function. Physiother Res Int. 1996;1(1):30-40.

14. Lima PO, de Oliveira RR, de Moura Filho AG, Raposo MC, Costa LO, Laurentino GE. Reproducibility of the pressure biofeedback unit in measuring transversus abdominis muscle activity in patients with chronic nonspecific low back pain. J Bodyw Mov Ther. 2012;16(2):251-7.

15. Koh EK, Park KN, Jung DY. Effect of feedback techniques for lower back pain on gluteus maximus and oblique abdominal muscle activity and angle of pelvic rotation during the clam exercise. Phys Ther Sport. 2016;22:6-10.

16. Stamatakis E, Grunseit AC, Coombs N, Ding D, Chau JY, Phongsavan P, et al Associations between socio-economic position and sedentary behaviour in a large population sample of Australian middle and older-aged adults: the social, economic, and environmental factor (SEEF) study. Prev Med. 2014;63: 72-80.

17. Alley S, Wellens P, Schoeppe S, de Vries H, Rebar AL, Short CE, et al. Impact of increasing social media use on sitting time and body mass index. Health Promot J Austr. 2017;28(2):91-5.

18. Zhang S, Xu Y, Han X, Wu W, Tang Y, Wang C. Functional and morphological changes in the deep lumbar multifidus using electromyography and ultrasound. Sci Rep. 2018;8(1):6539.

19. Azevedo DC, Lauria AC, Pereira AR, Andrade GT, Ferreira ML, Ferreira PH, et al. Intraexaminer and Interexaminer reliability of pressure biofeedback unit for assessing Lumbopelvic stability during 6 lower limb movement tests. J Manip Physiol Ther. 2013;36(1):33-43. 
20. Konrad P. ABC of EMG - A Practical Introduction to Kinesiological Electromyography[M]. Version 1.0 ed. Noraxon INC. USA.2005 60.

21. Chiarotto A, Maxwell $\sqcup$, Ostelo RW, Boers M, Tugwell P, Terwee CB. Measurement properties of visual analogue scale, numeric rating scale, and pain severity subscale of the brief pain inventory in patients with low Back pain: a systematic review. J Pain. 2019;20(3):245-63.

22. Lue YJ, Hsieh CL, Huang MH, Lin GT, Lu YM. Development of a Chinese Version of the Oswestry Disability Index Version 2.1. Spine (Phila Pa 1976). 2008;33(21):2354-60

23. Ansari B, Bhati $P$, Singla D, Nazish N, Hussain ME. Lumbar muscle activation pattern during forward and backward walking in participants with and without chronic low Back pain: an Electromyographic study. J Chiropr Med. 2018;17(4):217-25.

24. Vleeming A, Schuenke MD, Danneels L, Willard FH. The functional coupling of the deep abdominal and paraspinal muscles: the effects of simulated paraspinal muscle contraction on force transfer to the middle and posterior layer of the thoracolumbar fascia. J Anat. 2014;225(4):447-62.

25. Xiao J. Muscle atrophy. Singapore: springer; 2018. Adv Exp Med Biol. 2018; 1088:437-61.

26. Hodges PW, Richardson CA. Contraction of the abdominal muscles associated with movement of the lower limb. Phys Ther. 1997;77(2):132-44.

27. Gildea JE, Hides JA, Hodges PW. Morphology of the abdominal muscles in ballet dancers with and without low back pain: a magnetic resonance imaging study. J Sci Med Sport. 2014;17(5):452-6.

28. Hides J, Stanton W, Mendis MD, Sexton M. The relationship of transversus abdominis and lumbar multifidus clinical muscle tests in patients with chronic low back pain. Man Ther. 2011;16(6):573-7.

29. França FR, Burke TN, Caffaro RR, Ramos LA, Marques AP. Effects of muscular stretching and segmental stabilization on functional disability and pain in patients with chronic low Back pain: a randomized, Controlled Trial. J Manipulative Physiol Ther. 2012;35(4):279-85.

30. Ramos LAV, França FJR, Callegari B, Burke TN, Magalhães MO, Marques AP. Are lumbar multifidus fatigue and transversus abdominis activation similar in patients with lumbar disc herniation and healthy controls? A case control study. Eur Spine J. 2016;25(5):1435-42.

31. McGregor A, McCarthy I, Hughes S. Motion characteristics of normal subjects and people with low back pain. Physiother. 1995;81:632-7.

32. Willard FH, Vleeming A, Schuenke MD, Danneels L, Schleip R. The thoracolumbar fascia: anatomy, function and clinical considerations. J Anat. 2012;221(6):507-36.

33. van Dieën $J H$, Selen $L P$, Cholewicki J. Trunk muscle activation in low-back pain patients, an analysis of the literature. J Electromyogr Kinesiol. 2003; 13(4):333-51.

\section{Publisher's Note}

Springer Nature remains neutral with regard to jurisdictional claims in published maps and institutional affiliations.

\section{Ready to submit your research? Choose BMC and benefit from:}

- fast, convenient online submission

- thorough peer review by experienced researchers in your field

- rapid publication on acceptance

- support for research data, including large and complex data types

- gold Open Access which fosters wider collaboration and increased citations

- maximum visibility for your research: over $100 \mathrm{M}$ website views per year

At BMC, research is always in progress.

Learn more biomedcentral.com/submissions 